EPJ Web of Conferences 75, 06013 (2014)

DOI: 10.1051/epjconf/ 20147506013

(C) Owned by the authors, published by EDP Sciences, 2014

\title{
A new cracks detection device for magnetic steels
}

\author{
E. Manios ${ }^{1, *}$ and M. Pissas ${ }^{1}$ \\ ${ }^{1}$ Institute of Advanced Materials, Physicochemical Processes, Nanotechnology \& Microsystems, NCSR "Demokritos", \\ Patriarhou Grigoriou \& Neapoleos Str. 153-10, Aghia Paraskevi, Athens, Greece
}

\begin{abstract}
A portable detecting device was developed for the magnetic detection of cracks existing in railway tracks. The device is equipped with permanent magnets, which produce a uniform magnetic field inside and near the surface of the magnetically soft steel of a railway track, and GMR sensors. Due to the high sensitivity of the GMR sensors (in variations of the tangential component of the magnetic field which is parallel to the direction of motion) the device is capable of producing voltage peaks or dips, for quite small deviations of the near-surface magnetic field from the uniform field of the magnets. Finite element numerical simulations showed that the tangential component of the magnetic field exhibits sharp peaks above cracks, due to the stray magnetic field produced by them. Laboratory measurements, made on pieces of railway tracks with cracks, reproduced qualitatively the results of simulations. They showed that the sensors (moving near cracks) produce sharp voltage peaks with magnitude that depends on the size of the cracks. Based on these measurements, we conclude that the developed device can successfully detect cracks and defects in railway tracks and give information on their size. The method can be generalised for detection of cracks in all magnetic steels.
\end{abstract}

\section{Introduction}

Railways are currently one of the prime modes of transportation and, as they are closely associated with intensive passenger and cargo conveyance, they own high risk in terms of potential loss of human life and destruction of assets $[1,2]$. Despite the fact that improved safety standards are constantly being introduced, the forces acting on a rail track - due to wheel-rail contact stresses (WRCS) - are quite large and frequently repeated and - combined with the high speeds of modern trains can result in the quick evolution of small defects and cracks (which constitute Rolling Contact Fatigue or RCF) into partial or complete rail track failure, which can cause catastrophic accidents such as train derailments [1,2]. Therefore, it is essential to be able to detect RCF in its early stages and observe the time evolution of it (before the cracks increase in number and/or grow in size and the rail track breaks), in order to apply the correct maintenance/replacement strategy [1,3].

The Non Destructive Evaluation (NDE) methods that have been extensively used by the rail industry for surface inspection of rail tracks and wheels and detection of any surface defects/cracks are visual examination (by trained personnel or by automated optical cameras) [4] and magnetic induction techniques (like eddy current sensing systems) [4-6]. Similarly, ultrasonic wave

*e-mail: manios@ims.demokritos.gr (corresponding author) techniques [4,6] have been extensively used for the detection of internal defects/cracks. Other, more recent NDE methods, are electromagnetic acoustic transducers [4], laser ultrasonics [4], ultrasonic guided waves [3,4,7], wavelet packet decomposition [2], and alternating current field measurement (ACFM) probes [4].

A different approach, for the detection of surface cracks in magnetized rail tracks (and generally in magnetic steels), is the use of magnetic field sensors. It is known from magnetostatics that when a permanent magnet or a magnetized object is cut, magnetic poles are produced [8]. If these magnetic poles become spatially separated, then a stray magnetic field is produced around them [8]. Consequently, it is expected that any kind of surface defects on magnetized steel should produce stray magnetic fields around them. These stray magnetic fields can be measured by the sensors. Hall sensors have already been tested successfully for the magnetic detection of cracks $[9,10]$.

In this work we used GMR sensors. They are an excellent choice, for use as sensors of the magnetic field changes [11-14], due to their high sensitivity [14] and large output signal level, combined with low cost, low power consumption, small physical size and proven capacity to produce quite accurate voltage output signals even at high-speed movements. Indeed, GMR sensors are being used in hard disk drive (HDD) industry as the basic part of the reading heads of modern HDD, where the rotation frequency of the plates is of the order of $10^{4}$

This is an Open Access article distributed under the terms of the Creative Commons Attribution License 2.0, which permits unrestricted use, distribution, and reproduction in any medium, provided the original work is properly cited. 

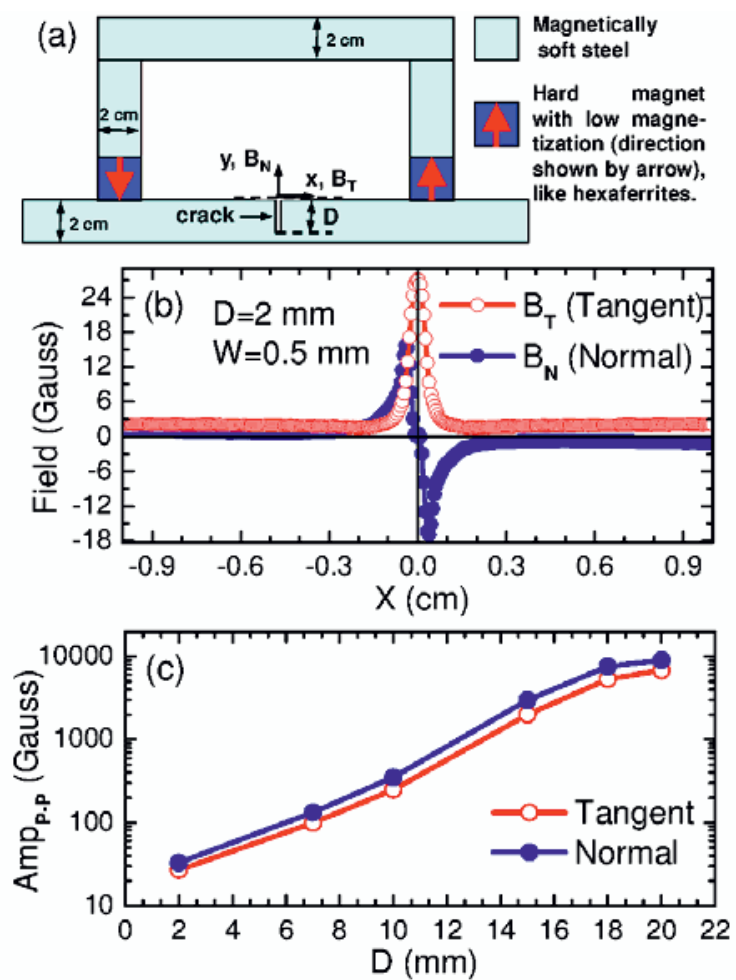

Fig. 1. (a) The simplified geometry used in the $2 \mathrm{D}$ numerical simulation of the stray magnetic field near a crack. The bottom (horizontal) rod represents the rail track, while the other parts represent the magnets configuration of the PMCDD. The depth of the crack is $D$ and its width is considered to be $W=0.5 \mathrm{~mm}$. (b) Components $\mathrm{B}_{\mathrm{T}}$ (tangential, empty circles) in the direction of motion and $\mathrm{B}_{\mathrm{N}}$ (normal, filled circles) of the magnetic field near the surface of the magnetized rail track, for different distances $\mathrm{X}$ from the crack. (c) Amplitudes (peak-to-peak, logarithmic scale) of $\mathrm{B}_{\mathrm{T}}(\mathrm{X})$ (empty circles) and $\mathrm{B}_{\mathrm{N}}(\mathrm{X})$ (filled circles) curves for various values of $\mathrm{D}$.

RPM. The GMR sensors were the basic part of the Portable Magnetic Crack Detecting Device (PMCDD), which was developed for the magnetic detection of cracks on the surface of rail tracks. Preliminary laboratory measurements, performed on rail tracks with known cracks, showed a correlation between the geometric characteristics of the cracks and the characteristics of the recorded signals.

\section{Device implementation}

Before proceeding with the development of the PMCDD, it was important to gain some insight on the behavior of stray magnetic fields near a crack that exists on the surface of a piece of magnetized steel. Since (for geometrical shapes with no special symmetries) it was not possible to solve analytically the equivalent magnetostatic problem, numerical methods were used (finite element numerical simulations with FEMM 4.2 [15]). Moreover, since the PMCDD would be based on GMR sensors that sweep the surface of a rail track and convert the changes of the in-plane component $\mathrm{B}_{\mathrm{T}}$ or the out-of-plane component $\mathrm{B}_{\mathrm{N}}$ of the stray magnetic field into changes of their output voltage $\mathrm{V}_{\text {OUT }}$, the numerical
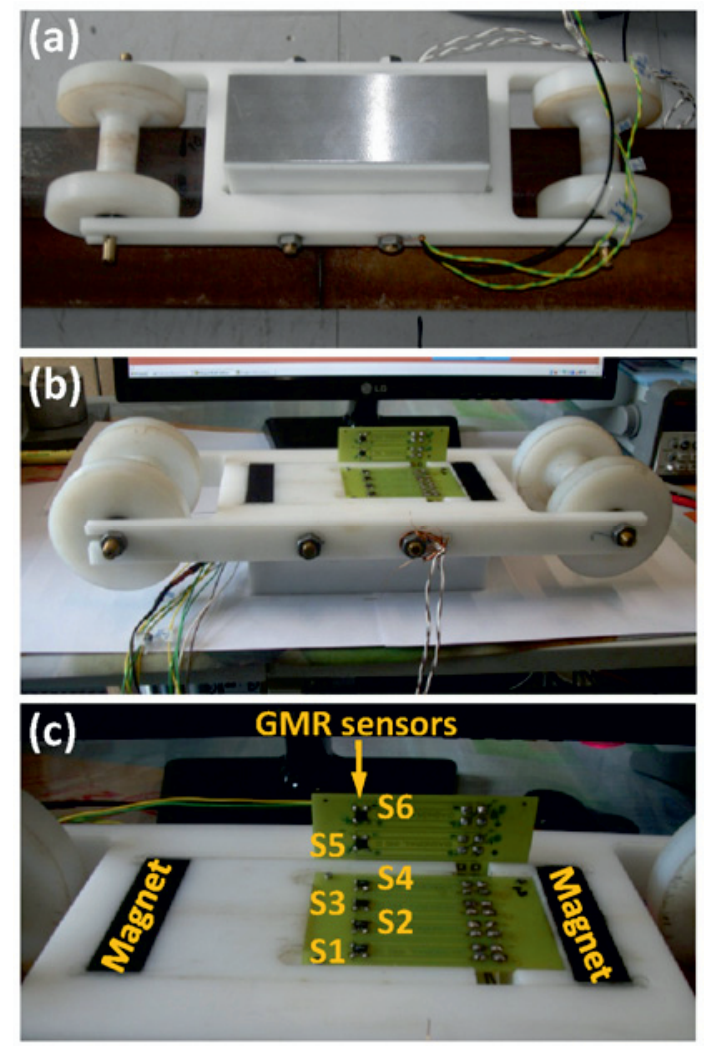

Fig. 2. (a) Top side view of the car which is used to carry the permanent magnets and the GMR sensors of the PMCDD. It is on a UIC 54 rail track. (b) Bottom side view of the car. (c) Closer view of the permanent magnets and the GMR sensors shown in picture (b). When the PMCDD moves on a rail track, sensors S1-S4 are horizontally oriented and closer to its head, while sensors S5, S6 are vertically oriented and closer to one of its sides. The magnetizations of the permanent magnets are vertical and oppositely oriented, as shown in figure 1(a).

simulations provided valuable information on the dependence of $\mathrm{B}_{\mathrm{T}}$ or $\mathrm{B}_{\mathrm{N}}$ on the position $\mathrm{X}$ of a surface site relative to the crack. This position dependence determines the expected form of the output voltage $\mathrm{V}_{\text {OUT }}(\mathrm{X})$ near a crack. In fact, the relation between $\mathrm{V}_{\text {OUT }}(\mathrm{X})$ and $\mathrm{B}_{\mathrm{T}}(\mathrm{X})$ or $\mathrm{B}_{\mathrm{N}}(\mathrm{X})$ is expected to be linear if the GMR sensors are not saturated. Figure 1(a) shows a simplified 2D geometry, which was used in the simulation. Figure 1(b) shows the position dependences $\mathrm{B}_{\mathrm{T}}(\mathrm{X})$ and $\mathrm{B}_{\mathrm{N}}(\mathrm{X})$ calculated for the crack of figure $1(\mathrm{a})$. $\mathrm{B}_{\mathrm{T}}(\mathrm{X})$ has the form of a sharp signal peak, with maximum at the middle of the crack and width of the order of the physical width $\mathrm{W}$ of the crack, while $\mathrm{B}_{\mathrm{N}}(\mathrm{X})$ behaves qualitatively in the same way as the derivative of $\mathrm{B}_{\mathrm{T}}(\mathrm{X})$. For larger crack depth $\mathrm{D}, \mathrm{B}_{\mathrm{T}}(\mathrm{X})$ and $\mathrm{B}_{\mathrm{N}}(\mathrm{X})$ have the same form. The width of the calculated signal peak is independent of the depth $\mathrm{D}$, while its amplitude increases drastically with D. In fact, figure 1(c) shows that the calculated dependence of amplitude from D is almost exponential.

The high sensitivity of GMR sensors makes them suitable for the magnetic detection of quite small surface cracks on magnetized steel and could probably also help us in the detection of under-the-surface cracks, by 
(a)
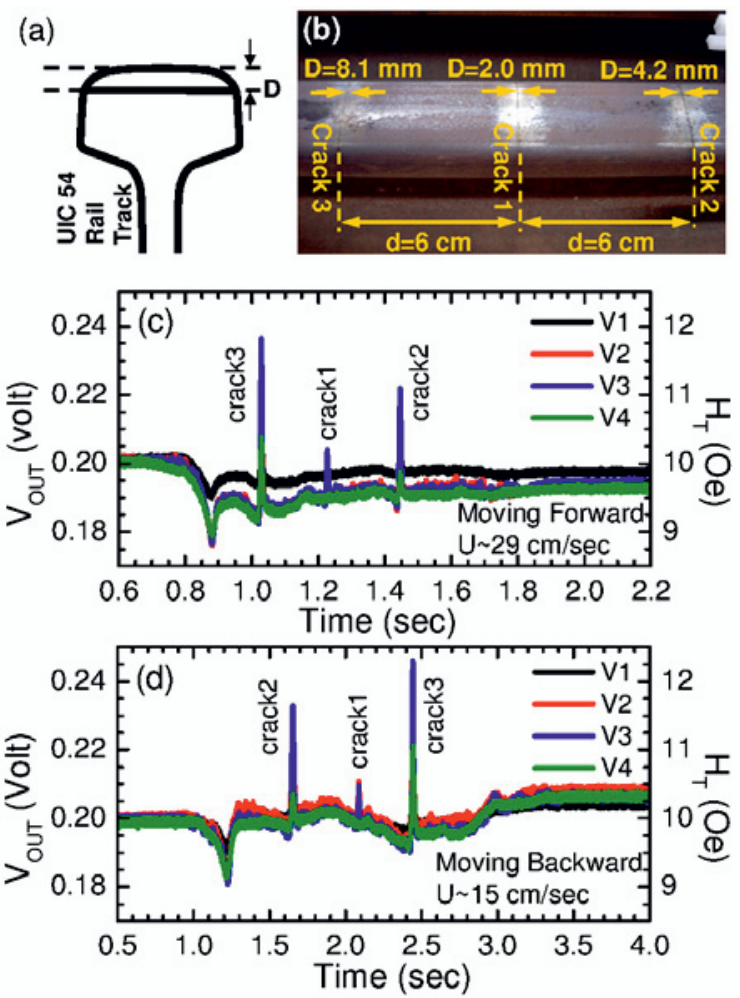

Fig. 3. (a) Schematic representation of a crack of depth D, which is perpendicular to the direction of motion on a rail track. (b) Pictures of three cracks of width $\mathrm{W}=0.3 \mathrm{~mm}$, created with an Electrical Discharge Machine (EDM). (c) Signals V1-V4 of the 4 GMR sensors S1-S4 for the case that the PMCDD was moving above these three cracks with constant speed U. The left axis shows the voltage output signals of the GMR sensors, while the right axis shows the tangential components $\mathrm{H}_{\mathrm{T}}$ of the stray magnetic fields. The bottom (horizontal) axis is left in time units. (d) Signals V1-V4 for movement of the PMCDD in the opposite direction.

measuring the anomalies produced in the uniform magnetic field just above the surface of magnetized steel. Also, their small physical size makes them suitable for the construction of arrays of sensors, which can give information on the spatial dependence of the measured magnetic field. The AA002-02 GMR sensors of NVE Corporation were used, which are sensitive to the $\mathrm{B}_{\mathrm{T}}$ component of the magnetic field. Their sensitivity is 4 $\mathrm{mV} \cdot \mathrm{V}^{-1} \cdot \mathrm{Oe}^{-1}$ and their saturation field is $15 \mathrm{Oe}$. Figure 2 shows the basic part of the prototype of the PMCDD, which is a car with the sensors, two barium ferrite $\left(\mathrm{BaFe}_{12} \mathrm{O}_{19}\right)$ permanent magnets for the magnetization of the rail track and pieces of soft magnetic steel. The combination of the pieces of steel, the barium ferrite magnets and the rail track behaves as closed magnetic circuit. The sensors were combined with a "NI USB-6216 BNC" data acquisition (DAQ) card of National Instruments, under the control of a LabVIEW based application (developed in our laboratory), running on a portable PC. The "NI USB-6216 BNC" DAQ card provides simultaneous real time acquisition of the analogue voltage output signals $\mathrm{V}_{\text {OUT }}$ produced by the sensors and can supply the DC bias voltage $V_{B}$ needed for their operation. Its maximum sampling frequency is

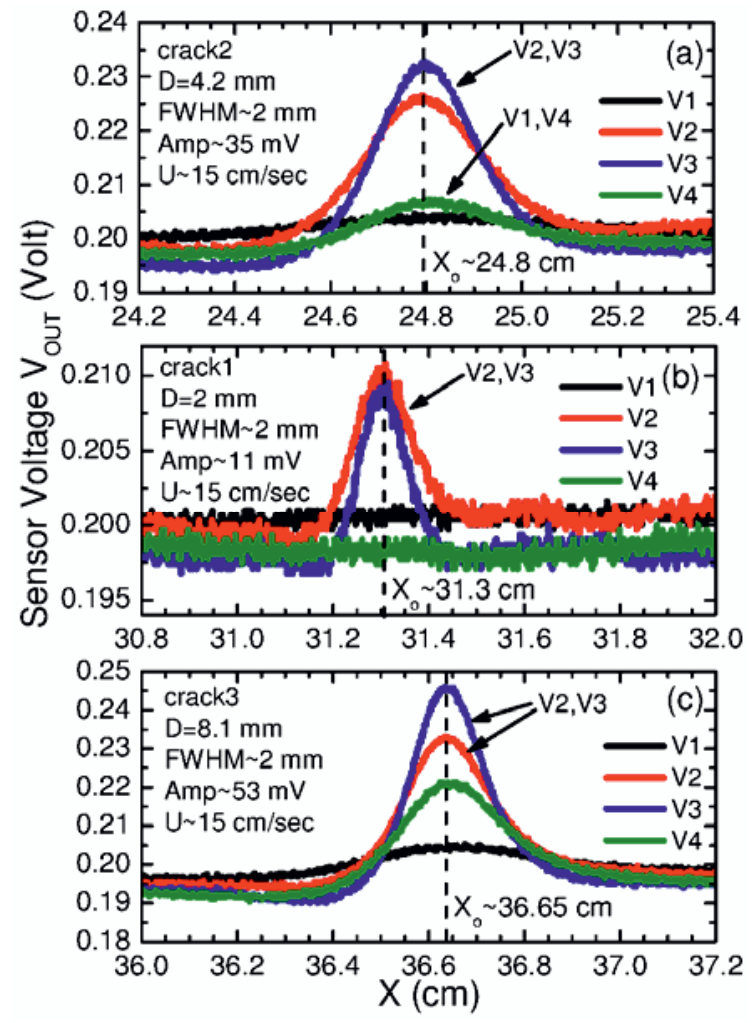

Fig. 4. Panels (a), (b) and (c) show in more detail the peaks of the signals of figure 3(d) corresponding to crack2, crack1 and crack3. The horizontal axes are in length units (the speed $U$ was used for the conversion). The approximate values of the center $\mathrm{X}_{0}$, peak amplitude Amp and FWHM (Full Width at Half Maximum) of the voltage peaks V2, V3 (signals of the 2 inner horizontally oriented sensors) are shown in each panel.

$400 \mathrm{kS} / \mathrm{sec}$ and it is capable of collecting and digitizing (16-bit representation) 8 different analog voltage signals.

\section{Laboratory testing}

The prototype of the PMCDD was tested on rail tracks with known cracks, in order to study the characteristics of the produced voltage signals and their correlation with the geometric characteristics of the cracks. The easiest way to have defects of known geometric characteristics was to deliberately produce them by using laboratory techniques. It should be noted that cracks produced on a rail track in this way are normally wider than the cracks naturally occurring in rail tracks due to their use. This was not a serious problem, since cracks with small width tend to produce more abrupt changes of the components of the magnetic field and therefore sharper and stronger peaks in the GMR sensor voltage signals. This means that if the sensors can detect an artificial crack produced by a cutting tool, then they can detect a naturally occurring crack with the same depth and smaller width. Figures 3-5 show representative results of these preliminary laboratory measurements, on pieces of a UIC 54 rail track. We note here that for the magnetic field in air it is $\mathbf{B}=\mathbf{H}$ and $1 \mathrm{Oe}=1$ Gauss. Therefore $\mathrm{B}_{\mathrm{T}}$ and $\mathrm{H}_{\mathrm{T}}$ shown in figures are equal. 


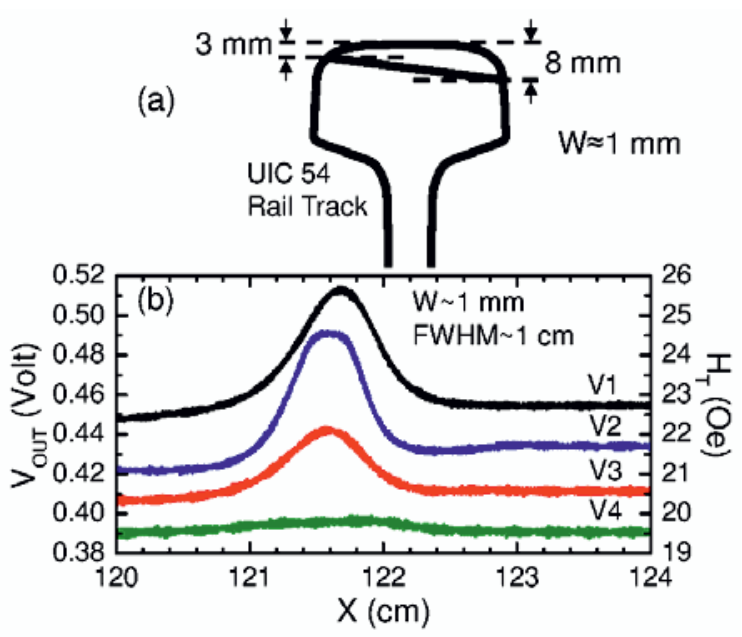

Fig. 5. (a) Schematic representation of a crack of varying depth and width $\mathrm{W} \approx 1 \mathrm{~mm}$, created with an electrical saw. (b) Signals V1-V4 of the 4 horizontal GMR sensors for the case that the PMCDD was moving above this crack. The left vertical axis shows the voltage output signals of the GMR sensors, while the right vertical axis shows the tangential components $\mathrm{H}_{T}$ of the stray magnetic fields. Signals V1-V3 have been shifted for clarity.

Figure 3 shows output signals of the 4 horizontally oriented GMR sensors which ware produced by moving the PMCDD on a rail track above three cracks created on its head by using an Electrical Discharge Machine (EDM). In all the recorded signals we observe a dip corresponding to the initial part of motion, when the device had to be accelerated from zero speed. This kind of signal dip is observed every time that the device is accelerated or decelerated and is probably a result of the secondary magnetic field produced by the eddy currents generated in the rail track steel due to magnetic induction. The presence of the 3 cracks produces 3 sharp peaks on the GMR output signals and the amplitude of a signal peak increases with the depth D of a crack, as it was expected from numerical simulations.

Figure 4 shows in more detail the output signal peaks of figure 3(d). In all cases, the amplitude of the signals $\mathrm{V} 2, \mathrm{~V} 3$ of the two inner horizontally oriented sensors is larger than the amplitude of the signals V1, V4 of the two outer horizontally oriented sensors (see figure 2(c) for sensors S1-S4), due to the curvature of the rail track head (see figure 4(a)). The accuracy in determining the positions of the cracks is of the order of the FWHM (Full Width at Half Maximum) of the peaks, which is of the order of $2 \mathrm{~mm}$. This value is one order of magnitude larger than the actual width $\mathrm{W}(0.3 \mathrm{~mm})$ of the cracks. This is not in agreement with the results of the numerical simulations previously discussed, where it was observed that $\mathrm{FWHM} \sim \mathrm{W}$, probably due to the purely static character of the magnetic field simulation. Another reason is the fact that the non-zero physical size of the GMR sensors, which affects the results of laboratory measurements, was not included in the magnetic field simulation. The amplitude of the output signal peaks increases drastically with the crack depth $\mathrm{D}$, as it was expected. The output signal peaks of figure 3(c) (not shown here) exhibit the same behavior. Finally, figure 5 shows the output signal peaks measured by the PMCDD above a crack of small but drastically varying depth D. The increment of $\mathrm{D}$ from one side of the crack to the other resulted in an increment of the amplitude of the recorded voltage peak of the corresponding GMR sensor, as it was qualitatively expected from numerical simulations.

\section{Conclusions}

A PMCDD was developed successfully. Preliminary laboratory measurements made on rail tracks with known cracks on their surface, showed that the recorded signals can be - at least qualitatively - related in a consistent way to the geometric characteristics of these cracks and the speed of the movement. The main advantage of this device is that it is able to detect the position of cracks with high accuracy and that the produced signals are highly sensitive to the size of the cracks. The device is also capable of detecting accelerations or decelerations, which make the recorded signals more complicate but could possibly be used as extra information when it is not feasible to maintain its speed at a constant value. Under investigation is the capability of the PMCDD to: (a) provide accurate values of quantitative parameters that describe the geometry of detected cracks, (b) operate at high speeds, (c) detect under-the-surface cracks. With suitable modifications of the car, the method can be used for detection of cracks in all magnetic steels.

\section{Acknowledgements}

This work was supported by the FP7 SME 2010-1 project DIAGNO-RAIL Grant Agreement No 262207 of the EC and the DECORAIL Greek Synergasia project.

\section{References}

1. D.F. Cannon, et al., Fatigue Fract. Eng.Mater. Struct. 26, 865 (2003)

2. H.A. Toliyat, et al., IEEE Trans. Ind. Appl. 39, 1454 (2003)

3. P.W. Loveday, J. Nondestruct. Eval. 31, 303 (2012)

4. M.P. Papaelias, et al., Proc. Inst.Mech. Eng. F, J. Rail Rapid Transit 222, 367 (2008)

5. M.J. Nicholas, et al., Insight 48, 346 (2006)

6. R. Pohl, et al., NDT\&E International 37, 89 (2004)

7. F. Lanza di Scalea, et al., Insight 47, 1 (2005)

8. J.D. Jackson, Classical Electrodynamics (3rd ed., John Wiley \& Sons, Inc., 1999) 184-197

9. A. Oota, et al., Rev. Sci. Instrum. 70, 184 (1999)

10. M. Göktepe, Sensors and Actuators A 91, 70 (2001)

11. M.M. Baibich, et al., Phys. Rev. Lett. 61, 2472 (1988)

12. P. Grünberg, U.S. Patent 4,949,039 (1990)

13. B. Dieny, et al., J. Appl. Phys. 69, 4774 (1991)

14. J.M. Daughton, et al., IEEE Trans. Magn. 29, 2705 (1993)

15. FEMM: http://www.femm.info/wiki/HomePage 